\title{
TURISMO COMUNITARIO Y SU ESTUDIO: REFLEXIONES DESDE UN ENFOQUE CULTURAL PROPOSITIVO PARA EL DESARROLLO RURAL'.
}

\author{
Joseph Orlando Torres Obando
}

\begin{abstract}
"No existen países pequeños. La grandeza de un pueblo no se mide por el número de habitantes, como no se mide por la estatura la grandeza de un hombre” - Víctor Hugo.
\end{abstract}

\begin{abstract}
Resumen:
En este escrito se pretende proveer al lector de una discusión acerca de lo que es y conlleva el turismo comunitario, en comunidades rurales y semi-rurales de Nicaragua. Se presenta una discusión teórica basada en una experiencia de campo en Villa Chagüitillo (Sébaco-Matagalpa), contrastando lo encontrado con datos de investigación documental, con la finalidad de comprender, como las diferentes modalidades de turismo alternativo - turismo cultural y rural - representan el eje sustentador del turismo comunitario, el cual se complementa con otras actividades económicas, integrando a las diferentes unidades familiares en su proceso de producción, facilitando de esa manera el desarrollo rural.
\end{abstract}

Palabras clave: turismo comunitario, turismo cultural, turismo rural, desarrollo rural.

\begin{abstract}
:
This written pretends to provide a discussion about what is and involves community tourism, in rural and semi-rural communities of Nicaragua. A way to present a theoretical discussion based on field experience in Villa Chaguitillo; contrasting the findings with data from desk research to ultimately understand bow the various forms of alternative tourism - cultural and rural tourism - is the bub of the community tourism is based, in line with other economic activities and integrating the different bousebolds in its production process, facilitating rural development.
\end{abstract}

Keywords: commnity tourism, cultural tourism, rural tourism, rural development.

\section{Introducción}

No es de hoy, claro está, que ante el bombardeo del estilo de vida urbanizado hacia las zonas rurales de Nicaragua, y ante la tendencia de la diversificación de estrategias de subsistencia para la vida rural, hablar de turismo comunitario como alternativa económica se vuelva un tema relevante para las ciencias sociales como la Antropología, o bien cualquier otra ciencia que estudie el desarrollo rural en función del bienestar humano.

He entrado a la discusión de la cuestión turística en vista que el turismo, es un fenómeno económico que ya no está en manos solo de quienes manejan grandes capitales para edificar hoteles de lujo, o cualquier otra instalación dedicada al ocio y la recreación, sino que también está en manos de comunidades campesinas y semi-rurales, aportando una nueva herramienta para las mismas (Cañada, 2010).

El propósito de este ensayo es establecer una herramienta teórico-metodológica que facilite el estudio de los elementos culturales aprovechables para el turismo comunitario; la elaboración de estrategias de clasificación que brinden pautas para la formulación de propuestas de desarrollo rural.

\section{Metodología}

Haciendo una revisión somera de la metodología utilizada para concebir este escrito, cabe especificar que se parte de una investigación antropológica cuya centralidad está en la recolección de datos

1. Dedicado a la población de Chagüitillo que me albergaron y me dejaron conocerles. Un sublime agradecimiento a mi amigo y mentor Msc. José Ramón Velásquez por abrirme espacios, y un amoroso agradecimiento a Alejandra Rebeca Pérez y mis padres por estar siempre a mi lado. 
etnográficos. Para esta labor se hicieron entrevistas a profundidad y observación directa en el espacio sociocultural en estudio, durante el mes de mayo del 2014.

Lo que se presenta en este estudio, las reflexiones hechas fruto de la labor de contrastación de los datos conseguidos de la primera labor de campo en Villa Chagüitillo (faltan dos momentos de campo), con la teorización resultante de la exhaustiva investigación documental realizada acerca del tema en cuestión.

Dicho de manera sintética, se presenta una discusión teórica basada en los datos conseguidos, producto de la experiencia de una primera visita de campo. Es importante advertir que no debe de esperarse en el desarrollo del presente trabajo una etnografía como tal, sino reflexiones como se indicó arriba.

A continuación se hace un recorrido por los conceptos y corrientes teóricas utilizadas para el abordaje del tema. Luego se expone la herramienta teórico-metodológica creada con el fin estructurar un sistema de clasificación. Para finalizar con una ejemplificación de su uso en Chagüitillo.

\section{Resultados y discusión}

\section{Teoría}

A medida que el tiempo ha venido avanzando, el turismo se ha vuelto todo un fenómeno de estudio, a tal punto que se ha subdividido en diferentes modalidades de turismo, cada una albergando una forma y oferta particular, para su estudio y comprensión.

Para la antropología social, la modalidad del turismo cultural se ha convertido en un área de estudio y profundización. De esta forma ha venido surgiendo una sub rama de la ciencia denominada antropología del turismo.

Pero la variación o permanencia de los rasgos culturales en las zonas donde el turismo cultural es una realidad, no debe por ninguna razón, ser el campo limitado en el que se detenga el estudio antropológico.

No porque no sea válido lo que pueda resultar de tales estudios, sino todo lo contrario, tales resultados permiten que este enfoque o rama de la ciencia, avance a un segundo plano de propuestas pertinentes para el desarrollo de las comunidades.
En las comunidades rurales de Nicaragua, el desarrollo local es un tema de agenda dada la necesidad de mejora de la calidad de vida de sus habitantes. Por ello, al momento de hacer indagaciones en aspectos de las prácticas de vida en el campo (expresión de la ruralidad) con fine de contribuir al desarrollo, se hacen uso de modelos definidos.

Uno de estos modelos es la nueva ruralidad. Al referirnos a este concepto, o enfoque, se habla de: "precarización del empleo rural que provoca una reestructuración de las fuentes de ingreso económico, la articulación con complejos agroindustriales, revalorización del estilo de vida y cultura rural, y continuas migraciones" (campo-ciudad, ciudadcampo) (Suárez \& Acuña, 2008).

Al relacionar el turismo con la nueva ruralidad, no resulta complicado darse cuenta que el primero, en comunidades rurales, forma parte de la reestructuración de fuentes económicas, diversificando los procesos de producción. Al momento de estudiar esta reestructuración, bien puede ser desde la cultura, la cual choca con el dilema de clasificar el fenómeno como turismo cultural o turismo rural. Por ello se hará a continuación una revisión y puesta en contexto de ambos términos.

Con relación al concepto de turismo cultural, siguiendo las descripciones del término dadas por el Consejo Internacional de Sitios y Monumentos, se puede entender como: "un movimiento de personas esencialmente por un motivo cultural, tal como un viaje de estudios, representaciones artísticas, festivales u otros eventos culturales, visitas a lugares o monumentos, folklore, arte o peregrinación". (Santana, 2003, pág. 37).

Es claro que el concepto indica un uso de la identidad local en sí misma, y otros recursos materiales que giran de forma simbólica sobre los valores identitarios atractivos para el público (turistas). Contrario a esto, el concepto de turismo rural es concebido como:

Una nueva alternativa económica, complemento de su producción, que permite el uso de la capacidad instalada ociosa de los establecimientos, así como también la inclusión de la mano de obra familiar no ocupada en la producción tradicional, principalmente mujeres y jóvenes. (Román \& Ciccolella, 2009, pág. 9) A diferencia del concepto de turismo cultural, el turismo rural hace referencia no al uso de la identidad como atractivo, sino a la incorporación de las demás 
unidades domesticas y productivas diferenciadas en la práctica agrícola tradicional, para atender al turista en un espacio abierto, natural en el hogar (entendida como instalaciones ociosas o poco utilizadas), teniendo estos "visitantes" o huéspedes temporales, un contacto directo con el medio rural.

Haciendo un análisis con mayor detenimiento, se llega a la conclusión que ninguno de los dos términos son equivalentes. Eso no significa que sean contrarios, más bien de alguna manera pueden verse como "complementarios", porque en la acción turística se dan ambas (una más evidente que otra), dependiendo el perfil o estructura del sitio.

Esta situación dual, exige la búsqueda de un término que englobe a ambos para tomar las dos perspectivas de manera complementarias. Este nuevo término que los aglutina es el turismo comunitario: "Surge a partir de modalidades del turismo desarrolladas en el espacio rural, conocidas también por los términos turismo cultural o etnoturismo, ecoturismo y agroturismo". (Skewes, Henríquez, \& Pilquimán, 2012, pág. 76). Por tanto, para este escrito, debe entenderse al turismo comunitario como una alternativa económica que se vale del turismo cultural y rural. Ambos se engranan con los demás procesos productivos locales, tanto en su dinámica como a nivel organizativo, con un sentido de asociatividad o de cooperatividad, integrando a las familias y todas sus unidades domésticas.

De lo anterior, se pude señalar que este término más global, permite a la antropología del turismo, entrecruzarse con la antropología rural para llevar a cabo un análisis completo del estilo de vida en la cultura rural. De modo que se relacione con propuestas (análisis) de desarrollo donde se mida la capacidad de "oxigeno" que las iniciativas turísticas (vistas desde este nueva conceptualidad) tiene en la comunidad.

A partir de esta nueva perspectiva, se puede analizar las entradas de capital en cada una de las familias.

De igual manera, observar el uso local de los valores identitarios como recurso que los posiciona dentro del circuito turístico comunitario, y como una forma de resistir a la presión ejercida por las industrias, el mercado, y las expresiones de vida globalizada presentes en las comunidades.

Uno de los estudiosos del tema del turismo, es el antropólogo Agustín Santana Talavera, quien en su artículo Desarrollos y conflictos en torno al turismo rural: claves y dilemas desde la antropología social
(2002) propone el "turismo alternativo" como contenedor del turismo rural, turismo cultural y otras modalidades más.

La propuesta de Santana Talavera es interesante, pero considero que es más apropiado el uso del término "turismo comunitario" en lugar de alternativo. El primero, es más holístico, porque permite: describir, analizar y entender el fenómeno en comunidades rurales nicaragüenses, El segundo, si bien comprende ambas modalidades (turismo cultural y rural) las ve desligadas y no como complementarias.

El turismo comunitario, a diferencia de "turismo alternativo", refleja el sentir asociativo, o de cooperativismo, necesario para alcanzar un desarrollo menos excluyente. El "alternativo", aunque absorbe las distintas modalidades turísticas no las articula y no hace hincapié en una forma puntual de organizarse para alguna propuesta de desarrollo.

Es útil señalar que toda propuesta dirigida a lo rural debe hacerse con un enfoque territorial, en especial la de turismo comunitario. Un territorio es el producto de una construcción social que apoya su estructura en la identidad (o identidades) de sus habitantes, considerado como capital social (Pérez, 2010). Es por ello que los valores identitarios puede variar de un territorio a otro.

No hace falta decir, que además del enfoque territorial, para su sostenibilidad, debe ser la misma comunidad quien gestione los recursos con que cuenta. Son ellos quienes le dan significados o re significan a estos, dentro de un proceso permanente en el cual están involucrados.

En consideración a lo antes expuestos, el proyecto de investigación antropológica relacionada al tema de turismo comunitario, propone un sistema de clasificación de recursos comunitarios que ayuden a formular una propuesta turística para el desarrollo rural de la localidad en estudio. Se le de utilidad o no a esta clasificación, el estudio le da elementos a posteriores investigaciones acerca de los recursos con los que cuenta la localidad y cómo se están gestionando.

\section{Propuesta de clasificación de elementos culturales}

Los elementos que se toman en cuenta para elaborar la clasificación, son aquellos aprovechables para el turismo comunitario. Se divide el turismo comunitario 
en dos modalidades: turismo cultural y turismo rural. Ambas se subdividen en categorías.

Para la modalidad de turismo cultural las categorías formuladas son: "recursos naturales, arqueológicos y organización comunitaria" donde se identifican sitios, museos, hallazgos arqueológicos, zonas aptas para caminatas 0 actividades al aire libre, monumentos, teatros, y las formas de organización que posee la comunidad. Tienen que ver con "expresiones identitarias" que corresponde a los componentes de la religiosidad, arte, mitología, gastronomía y prácticas de vida cotidiana.

Para la modalidad de turismo rural, la categoría formulada están relacionadas con las "actividades productivas", alusivas a las actividades agrícolas, la ganadería, la apicultura, la pesca, labores de artesanía, etc. Es importante agregar que un elemento que conforman los recursos naturales o arqueológicos, o en los procesos productivos, puede tener una incidencia significativa, dentro de la construcción de la identidad local. No obstante, con la categorización presentada se puede analizar dichos elementos de manera ordenada y precisa.

De esta forma, para continuar la discución, se da a conocer una muestra del uso de la clasificación de elementos culturales propuesta. El ejemplo está basado en los usos que tienen en la comunidad de villa Chagüitillo.

\section{La experiencia en Chagüitillo}

Lo idóneo es comenzar ubicando la localidad de Villa Chagüitillo. Esta es una comunidad semi-rural dentro de la jurisdicción del municipio de Sébaco, el cual está ubicado en el Departamento de Matagalpa, sobre la ruta del café. Su ubicación es un punto a favor en la temática, pues la ruta del café es de las más conocidas dentro del turismo nicaragüenses. En adición, la comunidad posee fácil acceso por carretera y ella representa un territorio de cultura indígena.

Durante el primer trabajo de campo realizado en el contexto dado a conocer al inicio, se identificaron los elementos culturales aprovechables para el turismo comunitario. Se mencionarán tan solo los que faciliten el mantenimiento de la discusión. Los elementos clasificados en mención son los siguientes:

-Recursos naturales, arqueológicos: se cuenta con un museo precolombino, foco catalizador de visitantes, mayormente estudiantes que llegan por motivos académicos, quienes suelen llevar extranjeros en calidad de voluntarios de proyectos comunitarios, albergados por familias locales.

El museo abrió sus puertas en el año 2005 y funciona como difusor de la cultura e historia local. Es uno de los proyectos comunitarios sostenido con ayuda de colaboradores internacionales. La construcción de dicho museo se puede considera como una evidencia del desarrollo de la comunidad; se autogestiona, pero depende del apoyo o colaboración externa y de las mismas autoridades o asociaciones presente en el territorio.

Chagüitillo cuenta con dos santuarios indígenas dentro de su territorio: uno lleva por nombre "Santuario de los venados" y el segundo nombrado como "Salto del mico", comúnmente los locales suelen llamarle "Apamico". Estos santuarios albergan una gran cantidad de petroglifos, testimoniando con ello, la presencia de importantes grupos originarios de la zona. Los santuarios, a pesar de no contar con instalaciones propicias para el fomento turístico, son por sí mismos, un atractivo potencial dado que el número de visitantes se ha incrementado en los últimos años.

Los santuarios están siendo gestionados en la actualidad por medio del museo, encargada de llevar a los visitantes a los sitios donde están los petroglifos. En la actualidad un problema asedia estos sitios: el despale ha secado la corriente de agua de los ríos y quebradas. La población ha expresado su inconformidad con la situación. Por medio de la Asociación para el desarrollo de Chagüitillo (ADCH) están tratando de encontrar formas de encontrarle una solución a la problemática.

El factor ambiental debe ser tomado en cuenta al elaborarse una propuesta de promoción del turismo comunitario, incluyendo un plan de conservación. En Chagüitillo la idea de reforestar los santuarios y acondicionarlos para caminatas, está acorde a la realidad del sitio, dado que puede darle mayor impulso al turismo.

-Forma de organización comunitaria: existe una alianza entre diez familias locales con el museo precolombino. Cuando el museo trae visitantes, estos son ubicados con alguna de estas familias, y en el momento que el visitante se va, deja una valoración de la experiencia al museo, luego, le es comunicada a la familia, a modo de retroalimentación.

Turismo comunitario y su estudio: reflexiones desde un enfoque cultural... 
La organización formada por las familias de Chagüitillo, refleja un sentido de asociatividad, al colaborar en común y de la mano con ADCH.

Hablando sobre las expresiones identitarias de Chagüitillo, hay dos festividades institucionalizadas. La primera es de índole religiosa: la celebración de San Juan Bautista, la cual se lleva a cabo los 24 de junio de cada año. Esta festividad tiene una gran significación cultural, en la comunidad, al punto que ese día es feriado para todos los trabajadores locales.

La segunda es más de tipo histórico político, porque celebran la elevación de Chagüitillo a Villa, siendo el 28 de septiembre la fecha de los actos conmemorativos. Durante las dos festividades mencionadas, se crea un espacio de cohesión social que funciona como refuerzo de la identidad, atrayendo gente foránea que atraído por los rituales religioso y cívicos, participan de las actividades.

Identificar momentos o símbolos culturales que refuerzan la identidad de una comunidad es un aspecto necesario para ser tomado en cuenta en cualquier tipo de proyecto, en vista que la actividad turística suele tener incidencia en las prácticas e imaginarios colectivos de los habitantes de la localidad.

Existe coincidencia en pensar que las identidades no son inmutables o estáticas, sino dinámicas. Pese a ello, se debe de monitorear y/o promover (para no usar la palabra "proteger", que sugiere estatismo) prácticas y valores con criterios de unificación colectiva, sabiendo que los valores identitarios representan ejes de atracción turística. Se debe de establecer medidas que "mitiguen" los efectos.

Ya que se mencionó lo correspondiente a los recursos arqueológicos y las expresiones identitarias, se debe de hablar de los aspectos productivos.

Hay que estar consciente que en una comunidad surgen diversos procesos productivos. Puede que uno de ellos predomine sobre los otros, ó en el que la mayoría de la población se siente involucrada.

Para las comunidades rurales y semi-rurales estos procesos están ligados a la agricultura, pero no debe de olvidarse que en algunas comunidades puedan existir otros procesos de producción relacionados con rubros no ligados a la actividad agrícola (caso de producción alfarera u otras prácticas artesanales). De igual forma, el turismo representa un rubro adicional, nuevo, que complementa a los demás dentro del territorio, sin reemplazarlos.

En Chagüitillo, a pesar que la agricultura se ha ido industrializando poco a poco, aún no desaparece la tradición de los cultivos domésticos. Esta condición permite que las familias que cuentan con espacios, crean huertos donde siembran productos perecederos para el autoconsumo, adaptando las instalaciones de la casa y sus patios, para que el turista se involucre en este proceso productivo, permitiéndole compartir con la familia que los hospeda.

Hecho el recuento de algunos de los elementos culturales encontrados y clasificados, puede que surja la pregunta icómo se pueden operativizar?, en primera instancia, debe de verse como una herramienta para el análisis, de forma ordenada de la cultura, identificando problemáticas que obstaculicen el desarrollo turístico comunitario, y local en general del territorio.

Consecuentemente, con los elementos clasificados, se pueden hacer valoraciones acerca de potenciales del sirio o área en estudio, con el fin de estructurar una propuesta de desarrollo turístico comunitario.

Para elaborar una propuesta de turismo pertinente se debe realizar una valoración de potencialidades, de lo contrario se puede caer en el error de una visión incorrecta de los potenciales turísticos y provocar un sobredimensionamiento de los proyectos en planificación. (Blanco, 2008).

De la afirmación citada, se deduce que una propuesta que no haya hecho las debidas valoraciones, inmersas en la realidad territorial del contexto en cuestión, no será más que una propuesta al aire. Podría llegar a pensarse que hasta vacía. Esto indica que el conocer la realidad social y cultural, se vuelve una prioridad para hacer valoración pertinente de potencialidades.

Siguiendo con lo anterior, de los datos hasta el momento expuesto y con las consideraciones planteadas, hay que valorar cuáles pueden ser las implicaciones del turismo y su impacto social. El autor Marvin Blanco en su obra Guía para la elaboración del plan de desarrollo turístico de un territorio (2008) da a conocer algunas pautas de los posibles impactos de una propuesta turística: "El impacto al medio ambiente, impacto en la identidad local, impacto económico y cambios en la organización comunitaria debido al turismo". (Blanco, 2008). 
Estás variables de impacto deben ser observadas antes y después de todo proyecto, aplicado a la realidad de la comunidad de Chagüitillo. De los elementos expuestos se vería: impacto a nivel ambiental (un problema ya existente y que podría agravarse con la visita de turistas) y un impacto cultural, visto desde la identidad local. Sin duda habría también un impacto económico por los ingresos que generaría el flujo de visitantes.

En adición, a medida que un rubro económico se articula con instituciones, empresas, erosionará las estructuras tradicionales de la comunidad. Las organizaciones comunitaria existentes se verán confrontadas o en tensión (Cañada, 2010), al haber desplazamiento de personas o nuevas reconfiguraciones a lo interno de la comunidad, en términos de vida material y de prácticas discursivas.

Esta oferta turística está fundamentada en el capital social rural con el que se cuenta. Es una actividad económica armonizada con las demás, e integradora de las unidades familiares involucradas, de manera directa o indirecta en la atención al visitante.

Dicha oferta puede ser analizada desde los recursos arqueológicos, las expresiones identitarias y los procesos productivos de la comunidad. Esta clasificación permite entender la realidad cultural y formular propuestas pertinentes de desarrollo rural enfocadas a completar el ingreso familiar por medio del turismo comunitario.

Esta propuesta de turismo incide en el desarrollo, porque además de atender la problemática económica, recoge de alguna manera otra problemática social que la población demanda. El caso de Villa Chagüitillo, que se abre paso dentro de la fiebre del turismo con un enfoque comunitario, puede retomar la idea inicial de reforestación de los santuarios indígenas para atacar el problema de la deforestación.
Es preciso mencionar el hecho que el turismo comunitario, con un fuerte carácter identitario y territorial, es una forma de resistencia a la presión ejercida por el mercado, la cultura de la "modernidad" y de la globalización. El turismo comunitario no es más que una parte de un abanico de herramientas para el desarrollo rural, de manera que, un territorio puede hacerle frente a la desigualdad social proponiendo iniciativas sobre este tema, sin ser afectados por las implicaciones que conlleva el proceso de adaptación y de gestión.

\section{Referencias bibliográficas}

Blanco, M. (2008). Guía para la elaboración del plan de desarrollo turístico de un territorio. San José, Costa Rica: PRODAR-IICA.

Cañada, E. (Coord) (2010) Turismo en Centroamérica, un nuevo escenario de conflictividad. Managua: Fundación Luciérnaga.

Pérez, S. (2010). El valor estratégico del turismo rural como alternativa sostenible de desarrollo territorial rural. Agronomía Colombiana , 507-513.

Román, M. F., \& Ciccolella, M. (2009). Turismo Rural En La Argentina. Concepto, Situación y Perspectivas. Buenos Aires.

Santana, A. (2002). Desarrollos y conflictos en torno al desarrollo rural: Claves y dilemas desde la antropología social. Tenerife: Universidad De La Laguna.

Santana, A. (2003). Turismo Cultural, Culturas Turísticas. Horizontes Antropológicos, Porto Alegre, 31-57.

Skewes, J. C., Henríquez, C., \& Pilquimán, M. (2012). Turismo comunitario de base comunitaria: Una experiencia alternativa de hospitalidad vivida en el mundo Mapuche. Talcao Sur De Chile. CULTUR Revista de Cultura e Turismo 73-85.

Suárez, N. d., \& Acuña, I. (2008). Lo Rural. Revista Facultad Nacional de Agronomía- Medellin , 4480-4495. 